\title{
Double tunnel technique reduces technical failure during POEM in cases with severe submucosal fibrosis $\square$
}

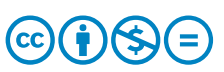

\author{
Authors \\ Nageshwar D. Reddy \\ Institution \\ Asian institute of Gastroenterology, Hyderabad, India
}

Zaheer Nabi, Mohan Ramchandani, Radhika Chavan, Jahangeer Basha, Manohar Reddy, Santosh Darisetty,

submitted 20.12.2020

accepted after revision 2.3.2021

Bibliography

Endosc Int Open 2021; 09: E1335-E1341

DOI 10.1055/a-1499-6817

ISSN 2364-3722

(C) 2021. The Author(s).

This is an open access article published by Thieme under the terms of the Creative Commons Attribution-NonDerivative-NonCommercial License, permitting copying and reproduction so long as the original work is given appropriate credit. Contents may not be used for commercial purposes, or adapted, remixed, transformed or built upon. (https://creativecommons.org/licenses/by-nc-nd/4.0/)

Georg Thieme Verlag KG, Rüdigerstraße 14,

70469 Stuttgart, Germany

Corresponding author

Zaheer Nabi, MD, DNB, 6-3-661, Asian Institute of

Gastroenterology, Hyderabad-500082, India

Fax: +91-40-23324255

zaheernabi1978@gmail.com

\section{ABSTRACT}

Background and study aims Submucosal fibrosis (SMF) is one of the major reasons for technical failure during peroral endoscopic myotomy (POEM). In this study, we aimed to evaluate the impact of double tunnel technique (DTPOEM) on the technical success of POEM in cases with severe SMF.

Patients and methods Data from patients with achalasia in whom SMF was encountered during POEM from Jan 2013 to Jan 2020 at our center were retrospectively evaluated. The technical success and adverse events (AEs) were analyzed and compared between cases with SMF who underwent POEM via single tunnel versus DT-POEM technique.

Results A total of 1,150 patients underwent POEM during the study period. There were 14 technical failures (1.2\%). SMF and severe SMF was found in 104 (9\%) and 21 (1.8\%) patients, respectively. The majority of patients $(17,80.9 \%)$ with severe SMF had type I achalasia. Overall, technical failures occurred in 14 cases (1.2\%) and severe SMF resulted in the majority of the technical failures $(10,71.4 \%)$. DT-POEM was utilized in 11 cases $(52.4 \%$ ) with severe SMF. The median procedure time with DT-POEM was 150 minutes (85210). There were no major AEs and DT-POEM was technically successful in all the patients. The technical success of POEM in cases with SMF improved after the adoption of the double tunnel technique ( $98.4 \%$ vs $68.3 \%, P=0.0001$ ). Conclusions Severe SMF is the most common reason for technical failure during POEM. DT-POEM improves the technical success rates of POEM in patients with severe SMF.

\section{Introduction}

Per-oral endoscopic myotomy (POEM) has emerged as a safe and efficacious treatment for the management of various esophageal motility disorders [1-3]. The technical success of POEM is high and reported in upto $90-100 \%$ cases. However, severe submucosal fibrosis (SMF) may pose special challenges during submucosal tunneling procedures like POEM [4, 5]. SMF has been shown to correlate with a prolonged operation, longer hospital stay, and higher perioperative adverse events
[5-7]. There is limited data regarding the outcomes of POEM in cases with SMF as well as techniques to improve technical success in these cases.

In this study, we evaluated the impact of double tunnel technique (DT POEM) on the technical success of POEM in cases with SMF. 

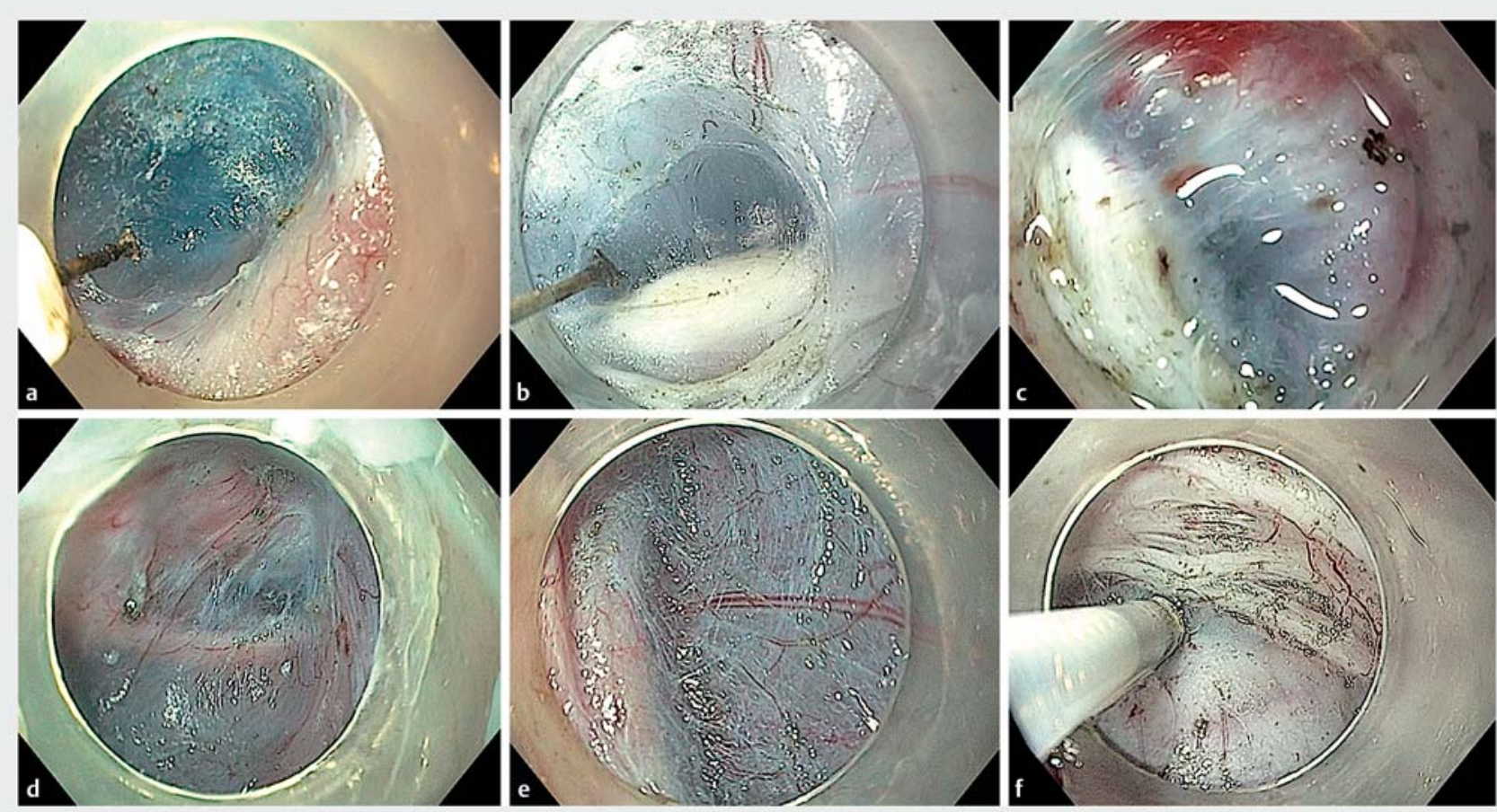

Fig. 1 Submucosal tunneling in cases with varying severity of submucosal fibrosis (SMF). a Submucosal tunnelling in a case with no SMF. $\mathbf{b}, \mathbf{c}, \mathbf{d}$ SMF with loose adherence of mucosa and muscle layer. Note that POEM could be completed using the same tunnel. e, $\mathbf{f}$ Severe SMF with complete adherence of mucosa and muscle layer. double tunnel POEM was performed in these cases.

\section{Patients and methods}

The data of patients with achalasia who underwent POEM during the study period (Jan 2013 to Jan 2020) were analysed, retrospectively. The cases with SMF were included in the final analysis. SMF was defined as either difficulty in lifting mucosa or sparse submucosal fibers resulting in poor separation between mucosa and muscle layer during tunneling. SMF was further classified as severe if mucosa and muscle were completely adherent and did not separate with repeated injections resulting in either termination of the POEM procedure or requiring a second tunnel for completion of POEM ( $\triangleright$ Fig. 1) [8]. The decision to abort POEM via first tunnel and create a new tunnel was made after agreement among at least two of the three POEM experts (ZA, MR, DNR).

\section{POEM technique}

POEM was performed using single or DT technique in cases with SMF. Single tunnel POEM was performed using standard technique as described previously [4, 9]. In cases with a poor mucosal lift with initial injection, several attempts were made at different sites. The procedure was abandoned in cases with complete adherence of mucosa and muscle layer during submucosal tunneling in the single tunnel group. Whereas, a second tunnel was created along different orientation in esophagus in the DT technique group.

The technique of DT-POEM is as follows ( $\bullet$ Video 1 ). Initially the submucosal lifting injection was performed anteriorly (2 O'clock) or posteriorly (5 O'clock). Mucosal incision and sub-

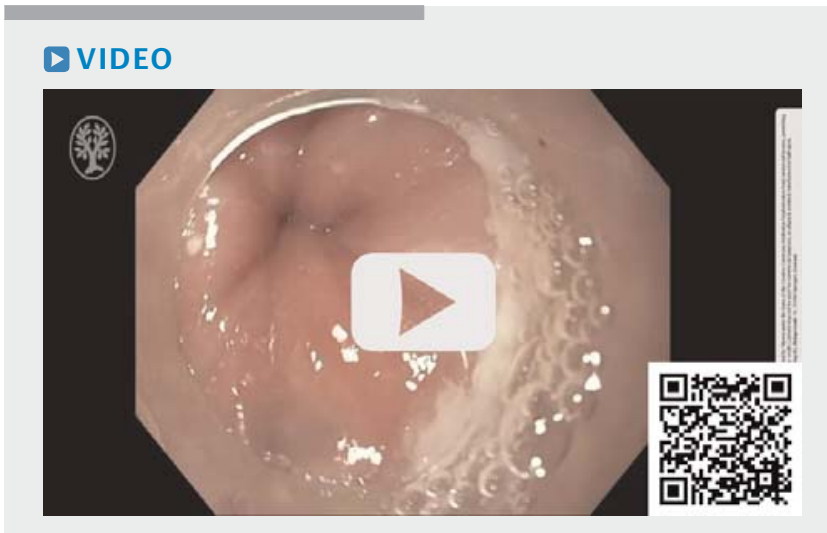

- Video 1 Double tunnel per-oral endoscopic myotomy in a patient with severe submucosal fibrosis.

mucosal tunneling were performed in the standard fashion. In cases with severe SMF not allowing continuation of tunneling, a second tunnel was created along the anterior or posterior esophageal wall depending on the initial site of tunneling ( $>$ Fig.2). The site of second submucosal injection was selected at least 1 to $2 \mathrm{~cm}$ below the initial incision along the alternate route (anterior or posterior). Subsequent procedure was accomplished using the standard technique of POEM. Finally, mucosal incisions of both the tunnels were closed using endoscopic clips. 


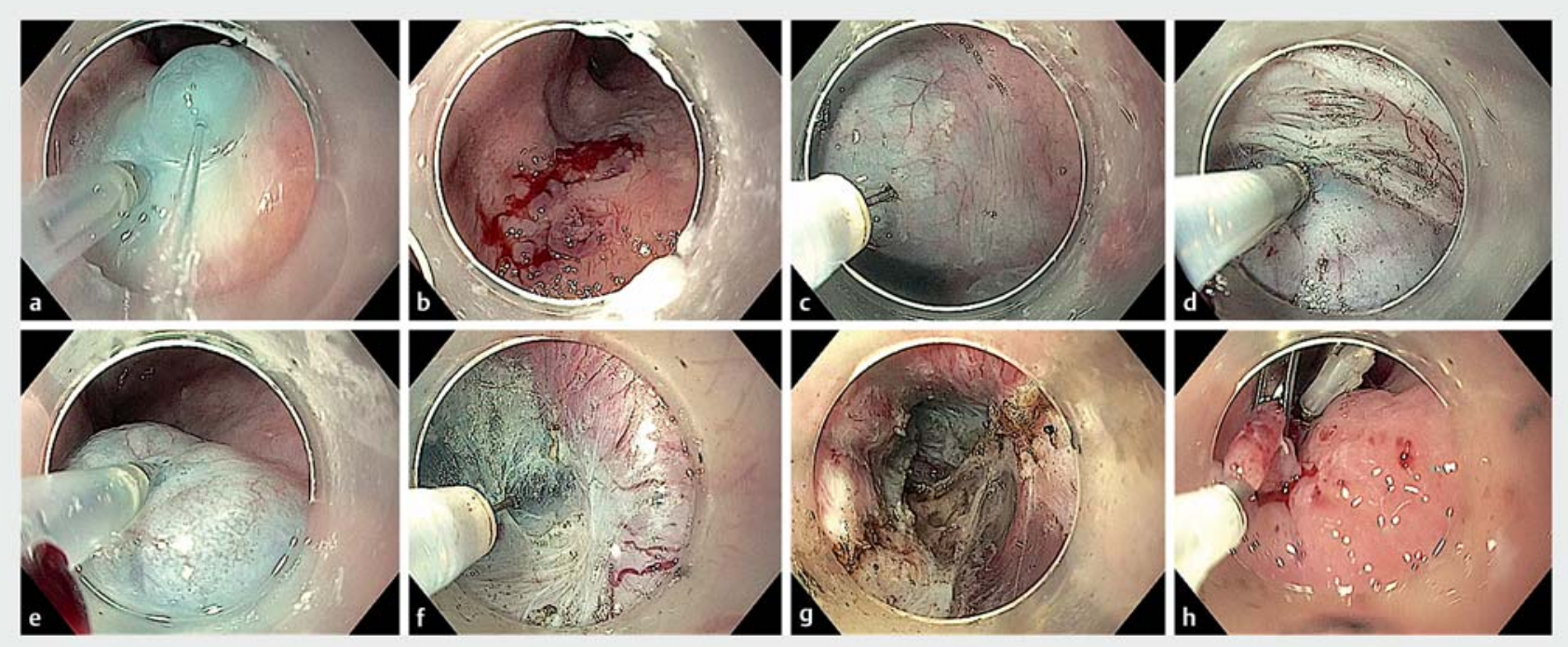

- Fig. 2 Technique of double tunnel per-oral endoscopic myotomy. a Submucosal injection along posterior esophageal wall (note the reflection of water jet indicative of severe submucosal fibrosis). b Poor lifting of mucosa after submucosal injection. $\mathbf{c}$ Adequate lifting of mucosa after injection along anterior esophageal wall. $\mathbf{d}$ Severe submucosal fibrosis after tunnelling for a few centimeters. e Third submucosal injection along the posterior esophageal wall about $2 \mathrm{~cm}$ below the first injection. $\mathbf{f}$ Adequate expansion of the submucosal space allowing for successful submucosal tunnelling. $\mathbf{g}$ Endoscopic myotomy performed after completion of tunnelling. $\mathbf{h}$ Closure of the mucosal incision using multiple endoclips.

\section{Primary outcome}

The primary outcome of the study was to evaluate the impact of DT-POEM on technical success in cases with severe SMF. The technical success of POEM procedure in cases with SMF was compared before and after the utilization of double tunnel technique.

\section{Adverse events}

Events requiring an additional procedure, such as needle drainage for capno-peritoneum, temporary cessation of procedure due to accumulation of retroperitoneal $\mathrm{CO}_{2}$, mucosal injuries requiring closure with endoclips, intra-procedural or post-procedural events leading to prolongation of hospital stay were considered as adverse events (AEs) [10, 11]. Major AEs were defined as those associated with hemodynamic instability, blood transfusion, mucosal injuries requiring special closure techniques, and major leaks [11].

\section{Statistical analysis}

The continuous data were expressed as mean (SD) or median (range) and the categorical data as frequencies unless otherwise specified. Comparison of categorical data between groups was performed using chi-square test with Yates correction. All the tests of significance were two tailed and a $P<0.05$ was considered to indicate statistical significance.

\section{Results}

A total of 1,150 POEM procedures were performed during the study period. Of these, POEM was performed using single tunnel technique in the initial cohort $(n=479)$ irrespective of the presence of SMF. Whereas, double tunnel technique was utilized as required in the latter cohort $(n=671)$. Overall, SMF of any severity was detected in $104(9 \%)$ patients (males 67, $51.82 \pm 10.57$ years) including $41(8.5 \%)$ and $63(9.4 \%)$ cases in the initial and latter cohort, respectively. The median disease duration was 48 (range $6-144$ ) months ( $\bullet$ Table 1 ).

Severe SMF was encountered in 21 patients $(1.8 \%$ ) (males $18,52.48 \pm 9.49$ years). The subtypes of achalasia in cases with severe SMF were type II (2), type I (17) and unknown (2). Prior treatment history and sigmoidization of esophagus were noted in nine $(42.8 \%)$ and five patients (23.8\%), respectively. POEM could not be completed in 14 patients $(1.2 \%)$ due to severe SMF in 10, inadvertent extension of mucosal incision in three, and circumferential leiomyoma leading to sigmoid achalasia in one. Mucosal edema or changes of stasis esophagitis were evident in 17 patients $(94.4 \%$ ) ( $\vee$ Table 2 , $\triangleright$ Fig. 3 ).

\section{Primary outcome}

Of 104 cases with SMF, POEM was successfully completed using single tunnel and double tunnel in 83 (79.8\%) and 11 patients $(10.6 \%)$, respectively. POEM could not be completed in 10 cases $(9.6 \%)$ due to severe SMF in the single tunnel group. DTPOEM technique was utilized in 11 patients (10.6\%) with severe SMF. The second tunnel was created along the posterior route (5 O'clock) in the majority of patients $(9,81.8 \%$ ) after failure to complete the initial tunnel via anterior route (2 O'clock). DT-POEM was successful in all the cases where utilized ( $\bullet$ Table 2). 
- Table 1 Procedure details in cases with SMF before and after utilization of the double tunnel technique

\begin{tabular}{|c|c|c|c|}
\hline & $\begin{array}{l}\text { Cohort } 1 \\
\mathrm{n}=479\end{array}$ & $\begin{array}{l}\text { Cohort } 2 \\
\mathrm{n}=671\end{array}$ & $P$ \\
\hline SMF (Overall) & $41(8.5 \%)$ & $63(9.4 \%)$ & 0.677 \\
\hline Severe SMF & $10(2.1 \%)$ & $11(1.6 \%)$ & 0.457 \\
\hline Technical success (SMF) & $28(68.3 \%)$ & $62(98.4 \%)$ & 0.0001 \\
\hline Technical failure & $\begin{array}{l}\text { Severe SMF (10) } \\
\text { Extension of incision ( } 3 \text { ) }\end{array}$ & Leiomyomatosis (1) & \\
\hline Orientation of POEM & Anterior 31 (75.6\%) & Anterior 27 (42.8\%) & 0.001 \\
\hline DT-POEM & 0 & $11(17.5 \%)$ & \\
\hline \multicolumn{4}{|l|}{ Mucosal edema } \\
\hline . SMF & $13(56.5 \%)^{1}$ & $25(65.8 \%)^{2}$ & 0.478 \\
\hline - Severe SMF & $7(70 \%)$ & $10(90.9 \%)$ & \\
\hline Disease duration months, mean (SD) & $\begin{array}{l}49.27 \pm 32.04 \\
\text { (range, } 9-120)\end{array}$ & $\begin{array}{l}53.70 \pm 33.96 \\
\text { (range, } 6-144)\end{array}$ & 0.508 \\
\hline Length of myotomy, cm & $n=28$ & $n=62$ & \\
\hline - Esophageal & $9.18 \pm 3.02$ & $7.36 \pm 2.18$ & 0.002 \\
\hline - Gastric & $2.90 \pm 0.54$ & $2.85 \pm 0.34$ & \\
\hline Procedure duration in minutes, mean (SD) & $79.44 \pm 27.92$ & $71.65 \pm 29.67$ & 0.184 \\
\hline \multicolumn{3}{|l|}{ Adverse events } & 0.605 \\
\hline - Mucosal injury & 4 & 5 & \\
\hline - Capno-peritoneum requiring drainage & 2 & 7 & \\
\hline - Submucosal hematoma & - & 1 & \\
\hline \multicolumn{4}{|c|}{$\begin{array}{l}\text { SMF, submucosal fibrosis; POEM, per-oral endoscopic myotomy; DT-POEM, double tunnel per-oral endoscopic myotomy } \\
{ }^{1} \text { Data not available for eight patients } \\
{ }^{2} \text { Data not available for } 13 \text { patients }\end{array}$} \\
\hline
\end{tabular}

\section{Double tunnel versus single tunnel POEM}

In cases with SMF, the technical success was achieved in 28 $(68.3 \%)$ in the initial cohort. The technical success of POEM procedure was significantly better after the adoption of the double tunnel technique in the latter cases with SMF $(98.4 \%$ vs $68.3 \%, P=0.0001)$. There was no technical failure due to severe SMF after adoption of the DT-POEM technique as compared to 10 failures in cases where the technique was not utilized ( $\triangleright$ Fig.4)

\section{Outcomes in technical failure cases}

Of the 14 cases with technical failures, four underwent POEM after a median of 3 months, six underwent pneumatic dilatation, two patients received botulinum toxin injection and two patients underwent esophagectomy for advanced achalasia and leiomyomatosis in one patient each.

\section{Adverse events}

There were no major AEs in patients with SMF who underwent POEM with or without DT-POEM technique. Minor AEs were encountered in $17(16.3 \%)$, including capno-peritoneum $(n=9)$, mucosal injury $(n=9)$, and submucosal hematoma $(n=1)$. The cases with capno-peritoneum were managed using needle decompression. Whereas, mucosal injuries were successfully closed using standard endoclips after the completion of procedure. Submucosal hematoma was noticed within the index tunnel in one patient who underwent POEM via the double tunnel technique. No intervention was required in this case as there were no signs of active bleeding ( $>$ Table 2 ).

\section{Discussion}

In this study, we found that severe SMF is rare but the most common reason for technical failure during POEM. DT-POEM is a safe and effective technique and reduces technical failure in patients with severe SMF.

SMF is one of the major reasons for technical failure during POEM $[5,8]$. The severity of SMF is relative and has not been well defined in the literature. Feng et al categorized SMF into three grades of severity - mild, moderate, and severe - based on the distribution or density of fibers and lifting effect of submucosal injection [8]. In their study, SMF was detected in all the cases, including mild in $73 \%$ and moderate to severe in $27 \%$ [8]. 
Table 2 Characteristics of patients with severe submucosal fibrosis.

\begin{tabular}{|l|l|}
\hline Patient characteristics & $\mathbf{N}=\mathbf{2 1}$ \\
\hline Severe submucosal fibrosis & $21(1.2 \%)$ \\
\hline Mean age in years (SD) & $52.48(9.49)$ \\
\hline Disease duration in months, median (range) & $66(18-144)$ \\
\hline Type I/II/III/unknown & $17 / 2 / 0 / 2$ \\
\hline Sigmoid achalasia & 5 \\
\hline Technical failures & $14(1.2 \%)$ \\
\hline Severe submucosal fibrosis & 10 \\
\hline Others & 4 \\
\hline Double tunnel technique & 11 \\
\hline Length of myotomy, cm & $4.64(1.36)$ \\
\hline - Esophageal & $3(0.67)$ \\
\hline - Gastric & $150(85-210)$ \\
\hline Procedure duration in minutes, median (range) & 3 \\
\hline Mucosal injury & \\
\hline
\end{tabular}

However, mild SMF usually does not pose special challenges to the POEM procedure as mucosa can be lifted easily. Moreover, it may be difficult to differentiate mild SMF from no SMF based on distribution and density of fibers. In our study, we utilized an objective and clinically relevant definition of SMF. Severe SMF was classified only if it precluded continuation of the POEM procedure, resulting in either abortion or requirement of a second tunnel to complete the procedure. With this definition, SMF and severe SMF were found in $9 \%$ and $1.8 \%$ of patients, respectively, who underwent POEM at our center.

The technical success in the entire cohort was $98.8 \%$. Overall, severe SMF accounted for technical failure in $0.9 \%$ of all cases. The majority (71.4\%) of the technical failures could be attributed to severe SMF. Our results are in concordance with previous studies where SMF resulted in technical failures in
$0.7 \%$ to $2.6 \%$ of cases and responsible for most (>90\%) of the technical failures $[5,8,12,13]$.

The risk factors for SMF include long disease duration ( $\geq 6$ years), sigmoid esophagus, mucosal edema, and prior interventions [5]. In our study, the median disease duration in patients with severe SMF was more than 5 years, the majority had type I achalasia and sigmoidization of esophagus apparent in nearly one-fourth of these cases suggestive of advanced achalasia. The presence of mucosal inflammation has been shown to correlate with the degree of SMF [8]. Although we did not grade the degree of mucosal edema, the changes of stasis esophagitis and mucosal inflammation were evident in most of the patients with severe SMF ( $>$ Fig. 3 ). Besides mucosal inflammation, the water jet sign, slow and uneven mucosal lift, and rapid dissipation of the bulge are other potential indicators of SMF ( $>$ Fig. 2).

The management options in patients with severe SMF include performing simultaneous tunneling and myotomy and open POEM and POEM via double tunnel technique [14,15]. The risk of mucosal injury may be higher with the former technique. Similarly, the safety concerns with open POEM preclude its widespread utilization [16]. DT-POEM is similar to the conventional POEM technique, and therefore, may not be technically difficult to perform. Sanaka et al reported the utility of creating a second submucosal tunnel in a patient with severe SMF [14]. Besides this report, there are no studies evaluating the role of DT-POEM in patients with severe SMF. Because the endoscopist has access to the entire esophagus, a second tunnel can be attempted at several different locations without much difficulty. In our study, the DT-POEM technique was successful in all the cases where utilized. Importantly, there were no technical failures due to severe SMF after the adoption of the DT-POEM technique.

Our results suggest that SMF is usually focal and a second tunnel along the opposite route allows for successful POEM in the majority of the cases [17]. On the other hand, one potential drawback of the technique is that the final length of the myotomy may be shorter than the initially intended length as the second tunnel is initiated 1 to $2 \mathrm{~cm}$ distal to the site of the initial tunnel. Nevertheless, a short esophageal myotomy has been shown to be adequate at least in type I and II achalasia [10,
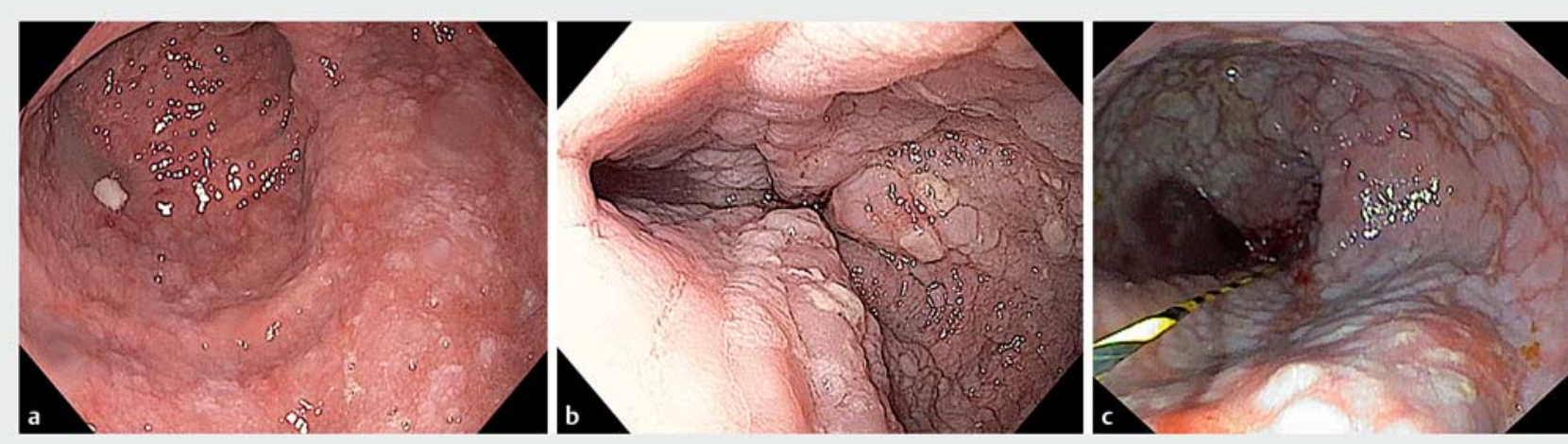

- Fig. 3 Mucosal changes in cases with severe SMF. a Mucosal inflammation obscuring the normal vascular pattern. b Severe inflammation and nodularity of the esophageal mucosa. c Mucosal inflammation with ulceration giving a cobblestone appearance to the mucosa. 


\section{POEM procedures $(n=1150)$}

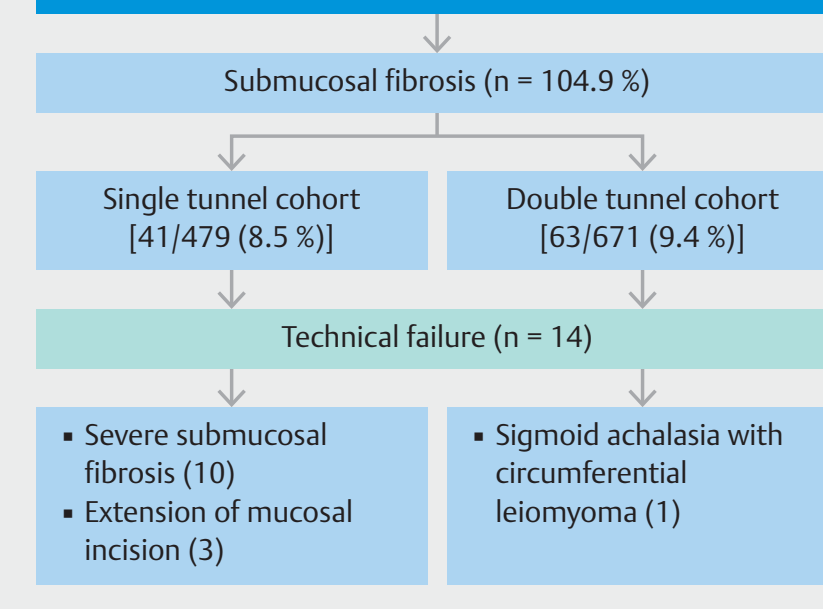

Fig.4 Graphic summary of the results of the study.

18-21]. The other drawback of the DT-POEM technique is the requirement for adequate expertise in performing POEM via both orientations (anterior and posterior).

There are several implications of our study. First, severe SMF is rare but the most important reason for technical failure during POEM. SMF is usually focal, and therefore, provides the opportunity of performing POEM by creating a second tunnel at a different site or orientation. In the present study, although mucosal changes were evident along both the anterior and posterior esophageal walls, a second submucosal tunnel could be created successfully. This finding suggests that mucosal changes may not correlate perfectly with the presence of SMF and the degree of SMF may vary along the esophagus, even with a similar grade of mucosal changes.

Our study has several strengths. To the best of our knowledge, this is the first study describing the utility of DT-POEM in patients with severe SMF. We acknowledge a few noteworthy limitations of our study. Technical success is dependent on operator's experience and improvisation in devices (e. g. water jetequipped knives). Therefore, improvement in technical success in the latter period may not be attributed solely to the DT-POEM technique. We used a stringent definition for defining SMF. Therefore, we may have underestimated the true incidence of SMF in the current study.

\section{Conclusions}

In conclusion, severe SMF is the major reason for technical failures during POEM. DT-POEM is safe and reduces technical failures in these cases.

\section{Competing interest}

The authors declare that they have no conflict of interest.
References

[1] Inoue $\mathrm{H}$, Sato $\mathrm{H}$, Ikeda $\mathrm{H}$ et al. Per-oral endoscopic myotomy: a series of 500 patients. J Am Coll Surg 2015; 221: 256-264

[2] Shiwaku H, Inoue H, Onimaru M et al. Multicenter collaborative retrospective evaluation of peroral endoscopic myotomy for esophageal achalasia: analysis of data from more than 1300 patients at eight facilities in Japan. Surg Endosc 2020; 34: 464-468

[3] Shiwaku H, Inoue $\mathrm{H}$, Sato $\mathrm{H}$ et al. Peroral endoscopic myotomy for achalasia: a prospective multicenter study in Japan. Gastrointest Endosc 2020; 91: 1037-1044 e1032

[4] Nabi Z, Ramchandani M, Chavan R et al. Peroral endoscopic myotomy in treatment-naive achalasia patients versus prior treatment failure cases. Endoscopy 2018; 50: 358-370

[5] Wu QN, Xu XY, Zhang XC et al. Submucosal fibrosis in achalasia patients is a rare cause of aborted peroral endoscopic myotomy procedures. Endoscopy 2017; 49: 736-744

[6] Wang Y, Liu ZQ, Xu MD et al. Clinical and endoscopic predictors for intraprocedural mucosal injury during per-oral endoscopic myotomy. Gastrointest Endosc 2019; 89: 769-778

[7] Xu S, Chai N, Tang X et al. Outcomes of peroral endoscopic myotomy in challenging achalasia patients: a long-term follow-up study. Surg Endosc 2020: doi:10.1007/s00464-020-07864-2

[8] Feng X, Linghu E, Chai $\mathrm{N}$ et al. New endoscopic classification of esophageal mucosa in achalasia: A predictor for submucosal fibrosis. Saudi J Gastroenterol 2018; 24: 122-128

[9] Ramchandani M, Nageshwar RD, Darisetty S et al. Peroral endoscopic myotomy for achalasia cardia: Treatment analysis and follow up of over 200 consecutive patients at a single center. Dig Endosc 2016; 28: $19-26$

[10] Nabi Z, Ramchandani M, Sayyed M et al. Comparison of short versus long esophageal myotomy in cases with idiopathic achalasia: a randomized controlled trial. J Neurogastroenterol Motil 2020: doi:10.5056/jnm20022

[11] Nabi Z, Reddy DN, Ramchandani M. Adverse events during and after per-oral endoscopic myotomy: prevention, diagnosis, and management. Gastrointest Endosc 2018; 87: 4-17

[12] Ngamruengphong S, Inoue H, Ujiki MB et al. Efficacy and Safety of peroral endoscopic myotomy for treatment of achalasia after failed Heller myotomy. Clin Gastroenterol Hepatol 2017; 15: 1531-1537 e1533

[13] Werner YB, von Renteln D, Noder T et al. Early adverse events of peroral endoscopic myotomy. Gastrointest Endosc 2017; 85: 708-718 e702

[14] Sanaka MR, Thota PN, Murthy SC et al. Creation of a second submucosal tunnel enabled successful per-oral endoscopic myotomy (POEM). J Thorac Cardiovasc Surg 2016; 151: e101-e102

[15] Li Y, LingHu E, Ding $\mathrm{H}$ et al. Peroral endoscopic myotomy with simultaneous submucosal and muscle dissection for achalasia with severe interlayer adhesions. Gastrointest Endosc 2016; 83: 651-652

[16] Liu W, Zeng XH, Yuan XL et al. Open peroral endoscopic myotomy for the treatment of achalasia: a case series of 82 cases. Dis Esophagus 2019; 32: 1-7

[17] Nabi Z, Reddy DN, Ramchandani M. Severe submucosal fibrosis - the “Achilles' heel" of peroral endoscopic myotomy. Endoscopy 2017; 49: 1116

[18] Gu L, Ouyang Z, Lv L et al. Safety and efficacy of peroral endoscopic myotomy with standard myotomy versus short myotomy for treatment-naive patients with type II achalasia: a prospective randomized trial. Gastrointest Endosc 2021; 93: 1304-1312 
[19] Wang ], Tan N, Xiao Y et al. Safety and efficacy of the modified peroral endoscopic myotomy with shorter myotomy for achalasia patients: a prospective study. Dis Esophagus 2015; 28: 720-727

[20] Li L, Chai N, Linghu E et al. Safety and efficacy of using a short tunnel versus a standard tunnel for peroral endoscopic myotomy for Ling type IIc and III achalasia: a retrospective study. Surg Endosc 2019; 33 : 1394-1402

[21] Huang S, Ren Y, Peng W et al. Peroral endoscopic shorter versus longer myotomy for the treatment of achalasia: a comparative retrospective study. Esophagus 2020; 17: 477-483 